\title{
Robotic Plugging Bolt Holes of Automobile Hub before the Spraying Process
}

\author{
Liang TONG ${ }^{1, \text { a }}$, Jing $\mathrm{LI}^{1, \mathrm{~b}^{*}}$, XiaoLu WANG ${ }^{1, \mathrm{c}}$ and Hui QIAN ${ }^{1, \mathrm{~d}}$ \\ ${ }^{1}$ School of Mechatronic Engineering and Automation, Shanghai Key Laboratory of Intelligent \\ Manufacturing and Robotics, Shanghai University, Shanghai 200072, China \\ atongl@shu.edu.cn, bian1982@shu.edu.cn, cwxlsgsg@qq.com, aianz@shu.edu.cn
}

\begin{abstract}
Keywords: automobile hub, tempo of plugging bolt holes, machine vision positioning, linear tracking Abstract. To realize the automation of plugging bolt holes for automobile hub before the spraying process, this paper builds an automatic plugging bolt holes system, which is based on industrial robot and integrates the visual positioning technology and linear tracking technology. And then the overall system solution of robotic automatic plugging bolt holes is given. For meeting the tempo requirement of plugging bolt holes for automobile hub before the spraying process, the tempo calculation models based on different gripper structures are built. Specific to the process of plugging bolt holes for automobile hubs with different number of bolt holes, the tempo of plugging bolt holes finished by gripper with different structures and suckers number is compared for choosing a gripper with optimal configuration and number of suckers. The visual technology is applied in the robotic plugging bolt holes, first, the flexible positioning of hub holes is finished by visual positioning technology. Then, the position tracking of the hub holes is realized by the linear tracking technology when the hub is moving on the conveyor. At last, the fast and accurate plugging bolt holes for automobile hubs based on industrial robot is completed. This system replaces the human to put plugs into hub bolt holes automatically, which enhances the automation level and productivity of the automobile hub spraying line.
\end{abstract}

\section{Introduction}

Automobile hub is an important part in a car. Spraying is the last working procedure before the manufacture of automobile hub is finished. The plugs should be put into the bolt holes of hub before spraying to prevent the bolt holes from being sprayed. The plugs being put into the bolt holes by human is a traditional way, which is inefficient, labour-intensive and easy to miss some holes.

In recent years, visual technology has been developed dramatically and been widely used in the industrial area, Such as automatic process, automatic assembly, workpiece detection [1,2]. Many Chinese universities and research institutions have studied the robotic intelligent application based on visual technology. For example, Yuliang Zhao and his colleague of Northeastern University have studied an online detection system of wheel hub based on visual technology. And the system can sort the style of wheel hub automatically [3]. Xinjian Fan and his colleague of Shandong University have designed a combined 2D-3D vision system for automatic robot picking. The system can be more efficient to process the picture and determine the spatial position of a target object [4]. However, these systems have just realized the function of visual position algorithm in the lab without considering the productive tempo in the real working condition. Ming Wang and his colleague of Hefei University of technology have analyzed the operating tempo of robot automation press line and then optimized the productive tempo of the system to enhance the productive efficient [5].

In this paper, we introduce a visual system composed of visual positioning technology, linear tracking technology and industrial robot technology, which can automatically put the plugs into the hub bolt holes on the moving conveyor. And In order to decrease the tempo of plugging bolt holes, the time that robotic grippers with different structure and different number of suckers put plugs into different number of automobile hub holes is compared and the optimal gripper is chosen. In conclusion we describe the system constitution and the workflow of plugging bolt holes in chapter 1 . In chapter 2 and 3, the tempo calculation models are created based on different type of gripper and then the most 
suitable gripper is chosen. Then the realization of visual positioning and linear tracking are illustrated in detail in chapter 4 .In chapter 5 , we present the conclusions.

\section{System Project Design}

According to actual working conditions, we configure the hardware composition of the system. The system is mainly composed of three units: industrial robot unit, image acquisition unit, integrated control unit. Then the principle and workflow of the system is introduced in this chapter.

The Constitution of Automobile Hub Plugging Bolt Holes System. As shown in Fig. 1, the system is composed of the hardware as follows: a fixed Fanuc M-2iA/3SL industrial robot with gripper for plugging bolt holes, a fixed Cognex camera for determining the number of automobile hub bolt holes, a fixed SONY XC-56 2D camera for locating the position of automobile hub bolt holes, a fixed camera for confirming that all bolt holes have been filled with plugs, a PC with image processing software, Fanuc R-30iB controller, an AA1000S encoder for calculating the moving distance of the automobile hub on the conveyor, a conveyor for moving automobile hub, a hopper for supplying the plugs etc.

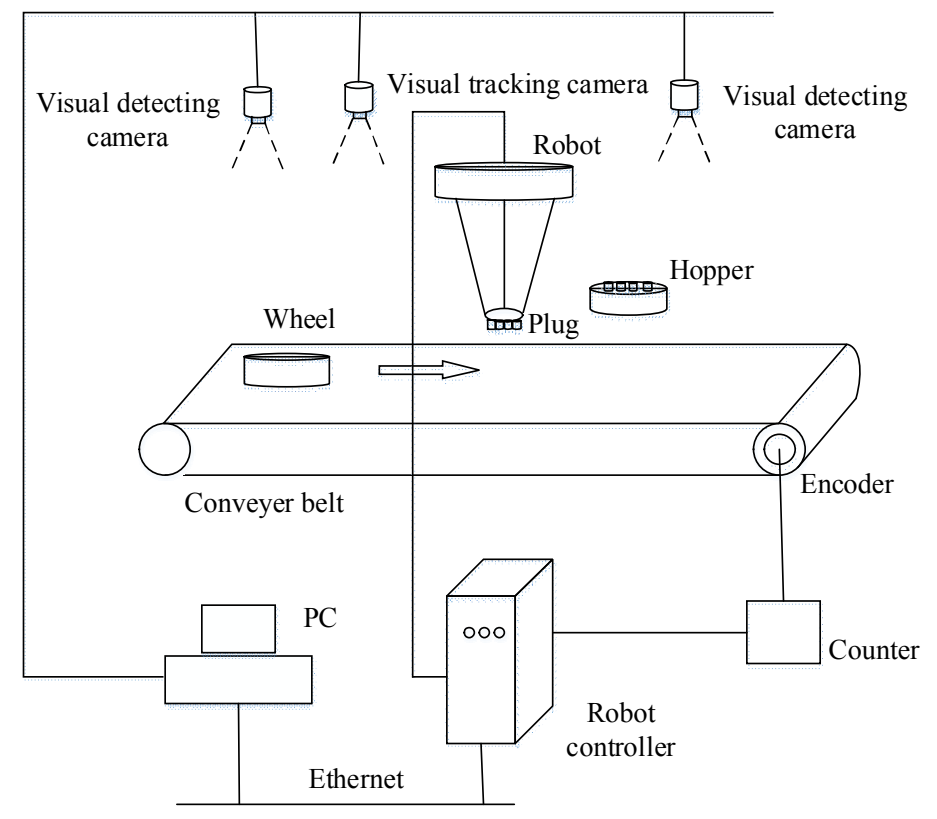

Fig. 1 System composition

The Workflow of Intelligent Robotic Plugging Bolt Holes. As shown in Fig. 2, the system operates as follows: (1) The automobile hub on the conveyor move to the work area, visual detecting camera starts to take photo. Then, the image is transferred to the PC visual software for image processing. Depending on the result of image processing, the number of automobile hub bolt holes can be determined. (2) After the number of automobile hub bolt holes being determined successfully, visual tracking camera starts to take photo. Then, the image is transferred to the visual software for image processing and getting the position information in the image coordinate. According to the relationships between image and world coordinate, the position information in the world coordinate can be calculated. Compared with the standard template, the relative position $\Delta \mathrm{X}, \Delta \mathrm{Y}, \Delta \mathrm{R}$ can be calculated. (3) When the wheel hub moves to the place position, the encoder will acquire the data $\Delta \mathrm{L}$ in $\mathrm{X}$ direction. According to $\Delta \mathrm{X}, \Delta \mathrm{Y}, \Delta \mathrm{R}$ and $\Delta \mathrm{L}$, industrial robot puts the plugs into the hub bolt holes. The hub will then be detected by visual detecting camera. If all holes have been filled with plugs, the robot returns to the origin for the next circle. 


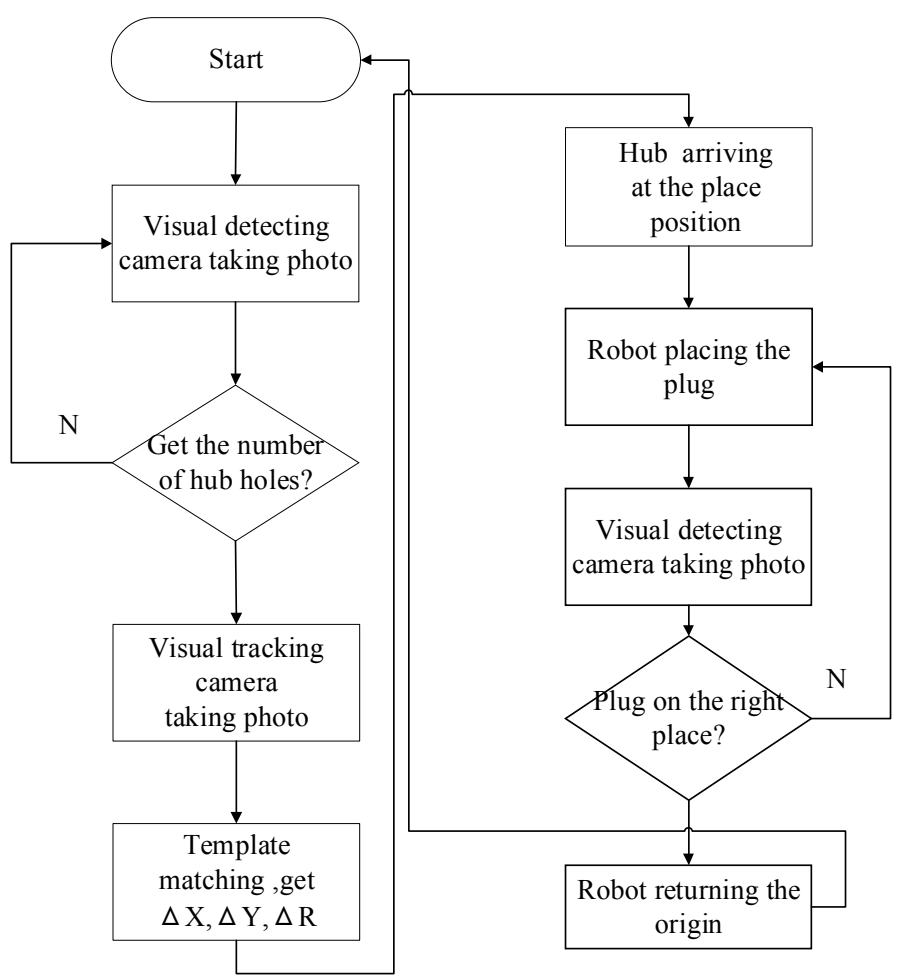

Fig. 2 System workflow

\section{The Tempo Calculation Model based on the Gripper Structure}

The gripper for plugging bolt holes is composed of air cylinders and suckers. And the air cylinder drives the sucker to realize the grabbing and putting of plugs. There are two typical gripper structures which differ in the arrangement forms of the suckers. The gripper shown in Fig. 3 is called linear gripper and shown in Fig. 4 is called circular gripper. Both of them can be configured with different number of air cylinders and suckers. There exist different plugging bolt holes tracks and plugging bolt holes time because the wheel hub bolt holes can be filled with plugs by the gripper with different structures and the number of suckers.

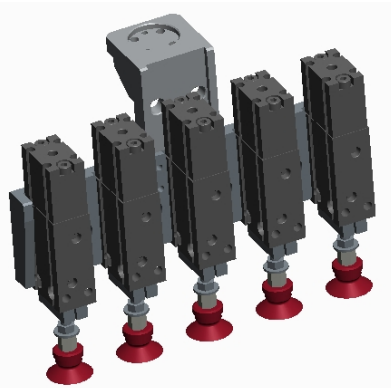

Fig. 3 Linear gripper

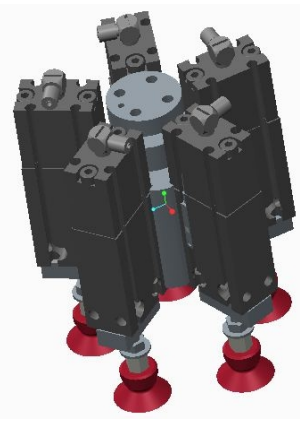

Fig. 4 Circular gripper

The Tempo Calculation Model based on Linear Gripper. As shown in the Fig. 5, the plugs are put into the automobile hub bolt holes by the linear gripper, which rotate around the wheel hub center position $\mathrm{O}$ with angular velocity $\omega$. The time that all bolt holes of an automobile hub are filled with plugs by linear gripper can be divided into three parts. The first part is the time that the gripper rotate around the wheel hub center position $\mathrm{O}$ with radius $\mathrm{R} 1$ for one circle. The second part is the time that gripper go back to the hopper for taking the plugs. If the number of suckers in the gripper is less than the number of the wheel hub bolt holes, the gripper have to go back to the hopper to get suckers again when the plugs in the gripper are used up. The third part is the time that gripper get down from a certain height to the position that the plug has been put into the hub holes correctly. 


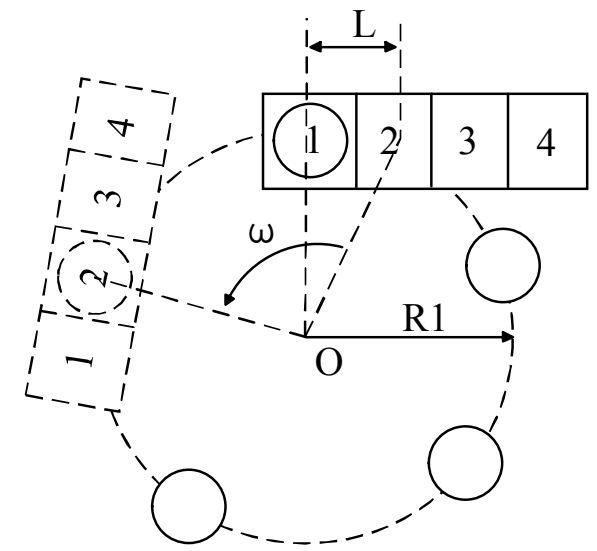

Fig. 5 Plugging bolt holes by linear gripper

Depending on the sequence of plugging bolt holes by linear gripper, the tempo calculation model of robotic plugging bolt holes is built.

$$
T_{L}=\frac{(Z-1)\left(\frac{360^{\circ}}{Z}+\arctan ^{-1}\left(\frac{L}{R_{1}}\right)\right)}{\omega}+(N-1) * K+Z * T .
$$

$\mathrm{Z}$ represents the number of wheel hub holes, $\omega$ represents the angular velocity that gripper rotate around the wheel hub center. $X$ represents the number of suckers in the gripper. $N$ represents the integer that $\mathrm{Z} / \mathrm{X}$ round up to. $\mathrm{K}$ represents the time that gripper go to hopper and back. $\mathrm{T}$ represents the time that gripper get down from a certain height to the position that the plug has been put into the hub holes correctly.

The Tempo Calculation Model based on Circular Gripper. As shown in the Fig. 6, the plugs are put into the automobile hub bolt holes by the circular gripper, which rotate around the wheel hub center position $\mathrm{O}$ with angular velocity $\omega$ and rotate with $\omega 1$ at the same time. The time that all bolt holes of an automobile hub are filled with plugs by circular gripper can also be divided into three parts. However, there exist different plugging bolt holes tracks between linear gripper and circular gripper, so the first part time is different.

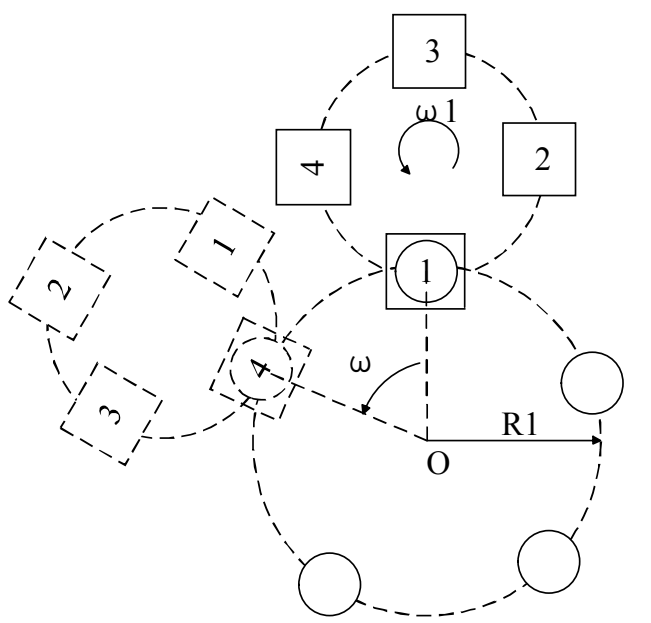

Fig. 6 Plugging bolt holes by circular gripper

Depending on the sequence of plugging bolt holes by circular gripper, the tempo calculation model of robotic plugging bolt holes is built.

$$
T_{C}=\frac{(Z-1) * \frac{360^{\circ}}{Z}}{\omega}+(N-1)^{*} K+Z * T .
$$


$\mathrm{Z}, \omega, \mathrm{X}, \mathrm{N}, \mathrm{K}, \mathrm{T}$ represent the same meaning in the chapter 2.1 .

\section{The Tempo Comparison between two Gripper Structure}

On the flexible spraying production line, there are four type of the wheel hubs with $4,5,6,8$ bolt holes respectively. The respective percentage of these four type of the wheel hubs is $10 \%, 20 \%, 50 \%, 20 \%$ and the daily output of the wheel hub is 10000 . The time that these four type of wheel hubs are filled with plugs by grippers with different structure and different number of suckers is compared for choosing the optimal gripper which keep balance between tempo of plugging bolt holes and system cost.

According to the tempo calculation Eq. 1 and Eq. 2 in the charpter2, the time that linear or circular gripper configured with 4,5,6,8 suckers puts plugs into 4,5,6,8 bolt holes of four type of wheel hubs can be calculated. The result is shown in the Fig. 7, from which we can get these following conclusion. (1) With the increase of wheel hub hole number, the time of plugging bolt holes increases gradually. (2) Under the condition that the number of suckers in gripper and the number of wheel hub bolt holes are confirmed, the time that the plugs are put into the wheel hub bolt holes by circular gripper is less than that by linear gripper. And with the number of holes increasing, the time deviation will get more serious. When the hub holes number is 8 , circular gripper can save $0.86 \mathrm{~s}$ in one plugging bolt holes than linear gripper or 1.74 hour in one day when the daily output of the wheel hub is 10000 . (3) When the number of suckers in the gripper is less than the holes number of the hub, the gripper have to go back to hopper to get the suckers again in the process of plugging bolt holes, which wastes more time. In this spraying line, the gripper with 8 suckers can save 0.5 hour in one day for plugging bolt holes than that with 6 suckers.

Circular gripper has faster tempo of plugging bolt holes than linear gripper, but it requires that the plugs on the hoppers should be arranged in a circle, which increase the complex of the feeding system design. The more suckers the gripper owns, the less time the plugging bolt holes takes. But it enhances the requirement of pneumatic system and control system. The tempo and cost of this productive line are considered comprehensively and the circular gripper with 6 suckers is chosen finally.

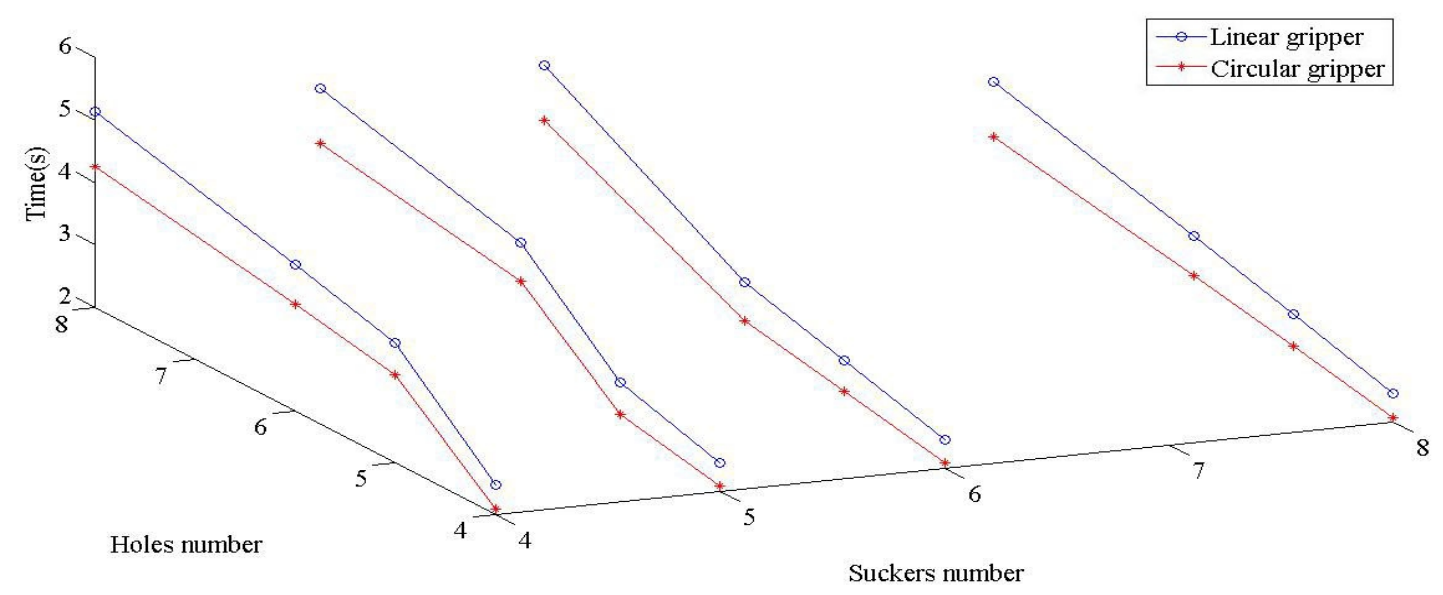

Fig. 7 One plugging bolt holes time

\section{The Realization of Robotic Automatic Plugging Bolt Holes based on Visual Technology}

In order to enhance the flexibility and productivity of robotic plugging bolt holes and realize the automation of plugging bolt holes system, the visual positioning technology is introduced to locate the position of wheel hub bolt holes and then the linear tracking technology is introduced to tracking the position of the wheel hub bolt holes on the moving conveyor. Prior to grasping the image of the automobile hub, the camera should be calibrated for creating the relation between image coordinate and world coordinate and getting the transform matrix [6]. The camera takes a photo for wheel hub and 
then the image of wheel hub is processed as follows: image pre-processing for smoothing the image and eliminating the interface noise [7], edge detecting and character recognizing for extracting contour of the wheel hub bolt holes [8] and getting the center position of the wheel hub in the image coordinate. The position of wheel hub bolt holes in the world coordinate can be obtained from the calculation between the transform matrix and the position of wheel hub bolt holes in the image coordinate. The encoder records the distance that the wheel hub moves in the time between twice plugging bolt holes. And then the distance data is transmitted to the robot controller which control the robot to put plug into the hub holes continuously.

Visual Position of the Hub Holes. Put a hub in the range of camera randomly, the camera takes a photo for getting the profile of the hub and the Fig. 8 shows that the hub holes have been recognized successfully. The hub image will be processed by the visual system and then the position of hub holes in the world coordinate will be calculated through the transformation of coordinate. The position of this hub will be taken as a reference position whose position deviation is zero and the program which conduct the robot to put plugs into the holes will be taken as standard program. Relative to the reference hub, the following hubs exist the positional deviation $\Delta \mathrm{X}, \Delta \mathrm{Y}, \Delta \mathrm{R}$ shown in the Fig. 9 because of the movement or the rotation of the hubs. According to $\Delta \mathrm{X}, \Delta \mathrm{Y}, \Delta \mathrm{R}$, the robot controller adjust the standard program and control the robot to put plugs into the hub holes.

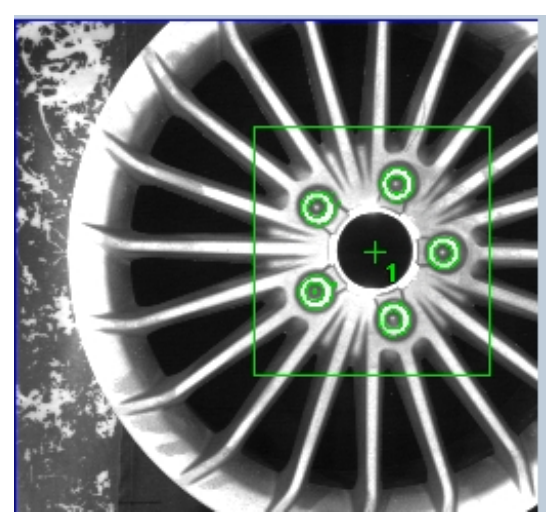

Fig. 8 Recognized the holes

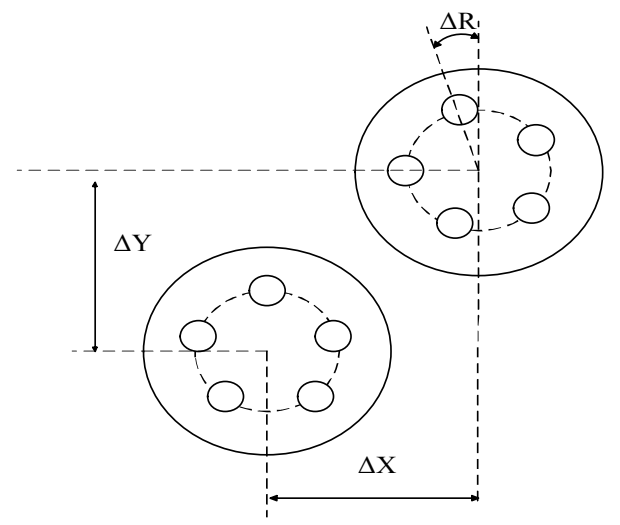

Fig. 9 Position deviation

Holes Tracking in the Plugging Bolt holes. In linear tracking system, the moving distance data of hub on the conveyor can be obtained by the encoder and transferred to the robot controller which control robot to put plugs into the moving hub in real time. As shown in the Fig. 10, the hub moves on the conveyor and the robot prepares to put the plug into the hub holes at the same time. When the hub arrive at the position for taking photo, the encoder records the pulse number of first relative position. And then the hub gets the position for first plugging bolt holes, the encoder records the pulse number of second relative position. The distance $\Delta \mathrm{L}$ that the hub moves from the position for taking photo to the position for first plugging bolt holes can be calculated according to the operation of the two pulse number. The $\Delta \mathrm{L} 1, \Delta \mathrm{L} 2, \Delta \mathrm{L} 3, \Delta \mathrm{L} 4$ can also be calculated in this way. The robot controller controls robot to put plugs into the hub holes according $\Delta \mathrm{L}, \Delta \mathrm{L} 1, \Delta \mathrm{L} 2, \Delta \mathrm{L} 3, \Delta \mathrm{L} 4$ continuously.

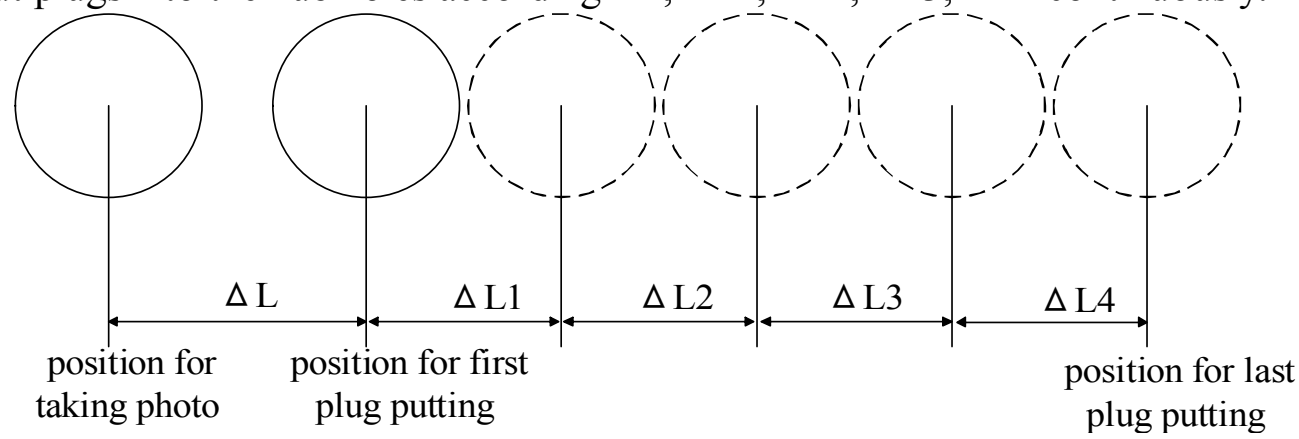

Fig. 10 Linear tracking 


\section{Conclusion}

This paper analysis the workflow and process of plugging bolt holes for automobile hubs on the spraying line of automobile hubs and then designs the overall solution of automatic system of plugging bolt holes for automobile hubs. Specific to the process of plugging bolt holes for automobile hub with different number of bolt holes, the different tempo of plugging bolt holes finished by gripper with different structures and sucker number is compared for choosing an optimal configuration scheme of gripper which is used to realize the automatic plugging bolt holes for automobile hubs as the end actuator of industrial robot. The vision positioning and linear tracking technology are applied to the robotic plugging bolt holes, which realize the automatic positioning and tracking positioning on the moving conveyor. This system replaces human to put plug into hub bolt holes, completes the fast and accurate plugging bolt holes for hub holes based on industrial robot and enhance the productivity of plugging bolt holes for automobile hub.

\section{Acknowledgment}

The research discussed in this paper is supported by Fanuc Robotics and the cooperation project of Shanghai Municipal Science and Technology Commission of Baoshan Districtunder Grant No. BKW2013107.

\section{References}

[1]Jianzhong Zhang, Yongyi He, Jun Li, Application research of vision-location in robotic assembly equipment, J. Journal of Mechanical \& Electrical Engineer. 8 (2011) 935-937. In Chinese.

[2]H. H. Shahabi, M. M. Ratnam, Prediction of surface roughness and dimensional deviation of workpiece in turning: a machine vision approach, J. Int J Adv Manuf Technol. 48 (2010) 213-226.

[3]Yuliang Zhao, Weijun Liu, Yongxian Liu, Renbo Xia, Research about a kind of automobile wheel classification system online, J. Machinery Design \& Manufacture. 10(2007) 164-166. In Chinese.

[4]Xinjian Fan, Xuelin Wang, Yongfei Xiao, A Combined 2D-3D Vision System for Automatic Robot Picking. Proceedings of the 2014 International Conference on Advanced Mechatronic Systems, Kumamoto, Japan, August 10-12. (2014) 513-516.

[5]Ming Wang, Ying Huang, Changrun Wang, The optimizations of productive time in the roboticized automation press line, J. Electronic Instrumentation Customer. 6(2008) 75-77. In Chinese.

[6]Z. Y. Zhang, A Flexible New Technique for Camera Calibration. IEEE Transactions On Pattern Analysis And Machine Intelligence. 11(2000)1330-1334.

[7]Zhenyu Liu, Bin Zhao, Haibo Zhu, Research of Sorting Technology based on Industrial Robot of Machine Vision. 2012 Fifth International Symposium on Computational Intelligence and Design. (2012) 57-61.

[8]Weibin Rong, Zhanjing Li, Wei Zhang and Lining Sun, An Improved Canny Edge Detection Algorithm. Proceedings of 2014 IEEE International Conference on Mechatronics and Automation August 3 - 6, Tianjin, China. (2014) 577-582. 